\title{
1 Towards the integration of niche and network theories
}

2 Oscar Godoy $^{1 *}$, Ignasi Bartomeus ${ }^{2}$, Rudolf P. Rohr ${ }^{3}$, Serguei Saavedra ${ }^{4}$

3 Instituto de Recursos Naturales y Agrobiología de Sevilla (IRNAS-CSIC) Av.

4 Reina Mercedes 10, E-41080 Sevilla, Spain

$5{ }^{2}$ Estación Biológica de Doñana (EBD-CSIC) Calle Américo Vespucio 26,

$6 \quad$ E-41092 Sevilla, Spain

$7{ }^{3}$ Department of Biology - Ecology and Evolution, University of Fribourg

8 Chemin du Musée 10, CH-1700 Fribourg, Switzerland

$9{ }^{4}$ Department of Civil and Environmental Engineering, MIT, 77

10 Massachusetts Av., 02139 Cambridge, MA, USA

$24 *$ To whom correspondence should be addressed. E-mail: ogodoy@irnas.csic.es 


\section{Abstract}

26 The quest for understanding how species interactions modulate diversity has progressed by

27 theoretical and empirical advances following niche and network theories. Yet, niche studies

28 have been limited to describe coexistence within tropic levels despite incorporating

29 information about multi-trophic interactions. Network approaches could address this limitation,

30 but they have ignored the structure of species interactions within trophic levels. Here we call

31 for the integration of niche and network theories to reach new frontiers of knowledge exploring

32 how interactions within and across trophic levels promote species coexistence. This integration

33 is possible due to the strong parallelisms in the historical development, ecological concepts,

34 and associated mathematical tools of both theories. We provide a guideline to integrate this

35 framework with observational and experimental studies.

36

37

$38 \quad$ Keywords

39 Coexistence, Feasibility, Multi-trophic Networks, Species interactions, Stability.

40

41 
43 One central aim in ecology is understanding how species interactions modulate biodiversity. At the origin of this interest is Darwin's legacy, who reasoned that species coexistence is less likely among closely related species as they tend to compete for similar resources for surviving explored the consequences of competition for a single-resource niche dimension $[4,5]$. However, researchers soon recognized that a species' niche is composed of multiple dimensions $[6,7]$. For instance, plants compete directly and indirectly for abiotic resources such as water, nutrients, and light [8-10], as well as for biotic resources in the form of mutualistic interactions (e.g., pollinators, disperses, and mycorrhizae) [11-14]. In addition to resource competition, parallel work has shown that antagonist interactions within a trophic level (i.e., intraguild predation) [15] as well as those coming from other trophic levels (e.g., predation, herbivory, and parasitism) are also part of a species' niche [16-19]. Moreover, positive interactions such as much more complex process than originally thought. multi-trophic interactions in coexistence by describing how competition within a guild of species is modulated by shared enemies (e.g., predators and pathogens). This concept set the path to recently recognize that competition for resources and predation can be of equal importance for limiting or promoting diversity within a guild of primary producers or 
consumers (e.g., plants or herbivores) [24, 25]. However, these advances together with niche studies are limited in their approach as their goal is to understand the role of species interactions in shaping species coexistence within one single trophic level [26]. The rest of species within a community that does not belong to the focal trophic level is considered to be always present and static. This critical limitation of niche studies clashes with the increasing interest of ecologists in disentangling the mechanisms maintaining species coexistence in more than one trophic level (see Figure 1 for a schematic representation). Indeed, part of this motivation is due to having multi-trophic information readily available $[27,28]$, but the fundamental question is how to extend niche theory to study the effects of species interactions on determining diversity across multiple trophic levels simultaneously.

To address this limitation, here we call for the integration of niche theory with network theory. Network theory has already partially addressed the challenge of how to consider the role of species interactions in shaping species coexistence across several trophic levels [29, 30], but it has missed the information of within trophic levels that niche theory incorporates. In particular, network studies have focused on the association of the structure of species interactions with community dynamics in mutualistic (e.g., plant-pollinator and plant-disperser) [31-35] and antagonistic systems (e.g., host-parasite, prey-predator, and plant-herbivore) [34, 36-38] Yet, because network studies emphasize species interactions between trophic levels, they consider that species within the same trophic level do not directly interact or they all interact with the same strength [33, 35].

Because niche studies lack the ability to describe species coexistence for more than one trophic level, and network studies ignore the structure of species interactions within trophic levels (Fig. 1), it is surprising that both theories have not spoken fluently to each other despite their complementarities can provide new research avenues and understanding of 
how species diversity is maintained. Our aim here is to show a direct integration of both theories as they share strong similitudes in their theoretical motivations, ecological concepts, and mathematical tools. This path of mutual understanding paves the road to combine theoretical concepts and associated toolboxes from both theories into a common methodological framework. We believe the emerging framework is particularly useful for investigating species coexistence in multi-trophic networks, which include competitive, mutualistic, and antagonistic interactions simultaneously. Additionally, we provide a road map that accommodates this new framework to experimental and observational studies.

\section{Conceptual parallelisms between niche and network theories}

Obtaining a common theoretical framework from the integration of both niche and network theories is straightforward as these studies have started from similar conceptual constructs, and after decades of research have independently converged on equivalent conclusions about the conditions leading to species coexistence. To reach the maximum audience, we verbally detail this historical convergence and explain here why both theories speak the same language despite using different technical terms. We also aim to present a rigorous mathematical explanation of this conceptual parallelism. This is possible because both theories use similar population dynamics models to build ecological theory rooted in the

Lotka-Volterra form [4, 24, 25, 35, 38-41] (Box 1). We are aware that the direct application of Lotka-Volterra models to describe natural systems might be limited because assume species linear responses, and do not take into account meta-community dynamics. Part of these limitations will be solved later when we will present more mechanistic models that capture additional nonlinear species responses in order to explain how to apply this emerging framework to experimental and observational approximations $[42,43]$. 
patterns of species distribution and co-occurrence within a trophic level based on how species

121 interact with the habitat they experience (Grinnellian niche), how they modify the habitat

122 (Eltonian niche) and how interact with other species in the community (Hutchinsonian niche)

123 [44]. Under classic niche theory, the only condition modulating species coexistence was the

124 amount of niche overlap between species [4, 45], which ecologists assumed to arise for instance

125 from differences in phenology, bill size, shade tolerance, or feeding preferences. The rationale

126 was that the smaller the niche overlap, the larger the chances of species coexistence [7, 46, $12747]$.

129 However, subsequent work [25, 41, 48] showed that niche differences alone are not

130 enough to determine species coexistence. Under recent advances of niche theory (also known

131 as "modern coexistence theory"), niche differences are only a stabilizing mechanism that tends

132 to promote coexistence when species limit themselves more than they limit others [48]. Modern

133 coexistence theory has provided techniques to directly measure niche differences as the relative

134 ratio between intra and interspecific competition [25], and consider that neutral dynamics occur

135 when species do not differ in their niches but have equivalent fitness [49]. The estimation of

136 niche differences using coexistence theory techniques remains phenomenological (i.e., the

137 source of variation is unknown), and recent studies are for instance mapping how species

138 functional trait differences relate to niche differences [50].

140 Conversely, species can also differ in their fitness. Fitness differences are related to

141 species' ability to capture and transform resources into offspring, which is generally a

142 combination of demographic parameters (e.g., fecundity, survival, and recruitment) and the

143 species' sensitivity to reduce these demographic parameters in the presence of neighbors

$144[25,43,49]$. Fitness differences in essence determine the superior competitor within a 145 species pair in the absence of niche differences. It has been well recognized that 
coexistence is the result of a balance between the relative strength of niche versus fitness differences. That is, two species will stably coexist when their niche differences overcome their fitness differences $[48,49]$ (see Table 1 for examples of both species differences across a wide range of organisms). This condition has also been reinterpreted as the larger the niche difference between two species, the larger the combination of their fitness differences compatible with their coexistence $[49,51]$ (Box 1). This reinterpretation is critical as it provides the main bridge of common understanding between niche and network theories explaining how species coexistence is possible.

Network research on species coexistence started by studying the stabilizing mechanisms for entire communities [5], rather than focusing on pairwise interactions. This stability was defined in a dynamical rather than a static way. Dynamical stability is the property of a system to return to an original equilibrium point (if it exists) after a pulse perturbation (e.g., a change in species abundances) coming from demographic stochasticity, which includes migration and random changes in birth and death processes. Early network studies showed that this dynamical stability depends on species interactions (analogous to niche differences) within and between trophic level compartments (contained in $\boldsymbol{\beta}$ matrix, Box 1). Importantly, a number of interesting questions emerged from these concepts, such as whether the observed structure of large multi-trophic systems necessarily leads to more dynamically stable communities [5]. However, extensive research showed that dynamical stability alone (as niche differences alone) is not enough to guarantee stable coexistence of all species in a community. This means that it can be possible to have a dynamically stable community where the equilibrium point will always lead to one or more species with zero abundance $\left(N_{i}^{*}=0\right)$, even if reintroduced into the community $[35,52,53]$. In other words, the system is dynamically stable but contains only a subset of species from the original pool. 
173 As it has happened with the historical development of niche theory, subsequent work on 174 network theory have shown that it is also necessary to account for the species' fitness in 175 order to evaluate the condition of whether species can attain positive abundances at 176 equilibrium [54]. Network studies called this condition feasibility, which also depends on 177 the species interactions contained in the matrix $(\boldsymbol{\beta})$ and the species' demographic

178 parameters $(r)[35,52,55]$ (Box 1). Importantly, these recent advances have shown that the 179 structure of species interactions between trophic levels can modulate the range of 180 combinations of demographic parameters leading to feasible systems [35, 55]. Therefore, 181 in line with niche theory, network studies also found that species coexistence within 182 communities depends on how the demography of species match the constraints imposed by 183 species interactions.

185 This historical convergence shows the existence of a common theoretical framework for 186 understanding how species interactions modulate diversity, which has two key ingredients:

187 1) species' demography and 2) the structure of species interactions. This structure is 188 contained in the $\boldsymbol{\beta}$ matrix described in Box 1. The take-home message of this framework is 189 that a community of species can coexist when both ingredients are combined in the 190 following way: species interactions define the coexistence space (i.e. the feasibility region) 191 and species coexist when the combination of their demographic parameters (i.e. fitness) 192 falls within this space (Box 2, Fig. 2). One crucial advantage of this framework is that it is 193 not limited to any particular type of multi-trophic interactions, and can be therefore 194 accommodated to both mutualistic and antagonistic interactions such as a plant-pollinator 195 or a predator-prey community. Another key important advantage is that this framework is 196 not either limited to two trophic levels. It can be extended to multi-trophic structures, 197 where three or more trophic levels are considered simultaneously. Indeed, these multi- 
trophic structures are simply the combination of competitive/facilitative interactions within trophic levels as well as antagonistic and mutualistic interactions between trophic levels [56] (Box 2).

\section{Coupling the integration of niche and network theories with}

\section{experimental and observational work}

204 We acknowledge that one critical step to consider in full this integrative framework

205 depends on how easily researchers can adapt it to their particular systems. The basic task is to obtain information of demographic parameters as well as species interaction coefficients within and between trophic levels. However, it is not so obvious how this information can be obtained and related to theory. We can start learning from the ability of recent advances in niche theory to couple theory with field and lab experiments [42, 57-60].

211 These studies suggest that the most rigorous way to proceed would be to conduct experiments in order to parameterize and validate a system of equations containing a model of population dynamics for each trophic level. Technically, this parameterization is

214 easier to obtain when the life-span between organisms is similar. In particular, population

215 models describing species dynamics with an annual life cycle seem among the best 216 approximations to choose for several reasons. They define the network structure and 217 species fitness in the exact same way as the original definition using the Lotka-Volterra 218 framework $[25,43]$, yet they are complex enough to include nonlinear mechanisms of 219 species coexistence such as the storage effect, and saturating functional responses to 220 competitive, mutualistic and antagonistic interactions (Box 3). They can also take into 221 account the effect of environmental variation in space and time on modifying diversity 222 maintenance due to changes in intransitive competition [59], intraspecific trait variation 
manipulate, models describing population dynamics have been successfully used for plants

$225[43,57]$, and can be extended to other annual organisms including pollinators (e.g., wild

226 bees), herbivores (e.g., snails, grasshoppers), or pathogens (e.g., fungal seed pathogens).

228 An alternative to experiments is the use of observational data (e.g., $[63,64]$ ).

229 Observational approaches are justified when organisms differ in their life-span, or when

230 their manipulation is not feasible for technical or conservation issues. The traditional

231 limitation of observational studies is that the structure of species interactions between

232 trophic levels is often easier to describe, at least at the species level, than the structure of

233 species interactions within trophic levels. This limitation can be solved by using

234 mathematical models fed with spatially explicit and/or temporal series data. These

235 methodologies allow inferring species demographic parameters and species interactions

236 from changes in species fitness due to both natural variations in the community density and

237 species relative abundances $[65,66]$. For example, recent work [64] combined statistical

238 models for survival, growth, and recruitment with individual-based models to describe

239 temporal pattern is plant species co-occurrences. These model-generated population

240 abundances were then integrated into projection models to estimate the structure of

241 competitive interactions within plant species.

243 Regardless of the approach selected, we stress the urgency of linking theory and empirical

244 work. We are at the dawn of understanding whether species characteristic, commonly

245 reported in the niche and network literature, are more strongly related to differences in

246 species demography or to the strength and sign of species interactions $[50,67,68]$.

247 Moreover, we are not aware of a single study that has attempted to empirically estimate in a

248 quantitative way the matrix of species interactions within and between trophic levels

249 simultaneously. We believe that taking such approach is crucial for answering an 
outstanding research question that emerges with the integration of both theories, namely,

251 how species interactions between trophic levels drive niche and fitness regions within

252 trophic levels and vice-versa. Therefore, this is the topic of our next section.

How do species interactions between trophic levels drive niche and fitness differences within trophic levels?

By coupling recent conceptual advances of niche and network theory, we are ready to understand how the species differences that determine coexistence within trophic levels (niche and fitness differences) feedback with the structure of species interactions that determine coexistence between trophic levels and vice-versa. To illustrate these ideas, let us consider a mutualistic plant-pollinator system (see graphical example in Fig. 2). What we have learned from prior work is that differences in feeding behavior, body mass, or insect phenology can contribute to the niche differences that tend to stabilize coexistence between plants (see Table 1) [69, 70]. However, pollinators also contribute to the fitness differences promoting plant competitive dominance. For instance, changes in the abundance of pollinators can, in turn, modify the competitive hierarchy of a plant guild by

266 increasing the number and the quality of seeds produced by pollinator-dependent plants.

267 Differentiating between these alternatives is crucial because if pollinators primarily drive 268 niche differences over fitness differences between plant competitors, then we can expect a more diverse plant community (e.g. $[11,70])$. A completely different outcome would occur

270 if pollinators primarily drive fitness differences among plants. In that case, a dominant 271 plant species favored by pollinators can dominate the community.

273 Similarly, considering pollinators beyond being a resource for plants implies that we have 274 to assess simultaneously their population dynamics. For instance, plant characteristics such 275 as floral morphology or plant phenological timing can contribute to the different pollinator 
requirements (i.e., niche differences) that stabilize their coexistence. But some plant species can also contribute to the dominance of a few pollinators (i.e., fitness differences) if those can particularly benefit from them as occur with pollinator specialists. All in all,

279 this could lead us to rethink if mutualistic interactions between trophic levels always

280 increase the likelihood of species coexistence. Traditionally, mutualisms have been

281 considered a positive interaction that enhance coexistence because the individuals involved

282 obtain a certain benefit that can be translated to their population growth rates (but see [16]

283 in a general context). However, to what extent these beneficial effects between particular

284 species across trophic levels can reduce the likelihood of species coexistence in the entire 285 system (i.e., within and among trophic levels) is not known yet (Fig. 2).

287 Note that we need to use a geometrical rather than an algebraic approach to study fitness 288 and niche differences for more than two species (see Fig. i in Box 2). This approach 289 informs us whether species coexistence is possible when the fitness differences between 290 species falls within the feasibility domain (Fig 2). Moreover, this approach allows us to 291 quantify how environmental variation modulates the extent of the feasibility domain and 292 the differences in fitness between species. Estimating these environmental-dependent 293 relationships is important as they determine how strongly can be an ecological community 294 perturbed without pushing species towards extinction. As a rule of thumb, the closest the 295 fitness differences to the edge of the feasibility domain, the lowest the ability of the 296 community to face perturbations (Fig. 2) [71]. It is also important to note that this 297 approach can be applied to other network types, such as food webs, parasitoid webs [24, 298 72], and multi-trophic networks combining antagonistic and mutualistic interactions [56, 299 73]. 
need a framework for describing species population dynamics as function of species demographic parameters and species interactions within and between trophic levels. For example, for a plant-pollinator system, this framework can be a system of two annual population models (one for each trophic level) that can include a storage effect component

306 if desired (Box 3). Second, in order to parameterize the models, we need information on species demography. For species demographic parameters, such as per capita growth rate in the absence of competition, germination rate, or larval survival, can be inferred relatively easy from experimental or observational data $[43,57,64,66]$. Third, we need to estimate

310 the matrix $\boldsymbol{\beta}$ that summarizes species interactions across trophic levels.

This third step is by far the most challenging aspect as the number of parameters that need to be estimated grows exponentially with the number of species in the community. In

314 principle, these estimates can be obtained from statistical models fitting empirical or 315 observational data $[27,59,74]$. For intra and interspecific competitive coefficients within 316 plants and within pollinators, these parameters can be obtained by describing how species 317 per capita growth rates depends on each competitor's relative abundance [50, 59] (Fig. ii 318 in Box 3). For the case of mutualistic effects of plants on pollinators and vice versa, the 319 procedure is similar to the one previously described, but this time per capita growth rates 320 should be described as a function of the relative abundance of each mutualistic species. In 321 the likely case that this option is not feasible, one possibility is to group species by 322 functional groups, and estimate interaction coefficients (at that resolution) via changes in population size of both trophic levels through time [75]. While the functional-group approach assumes uniformity of responses within functional groups, it might be a 325 requirement when scaling up to higher dimensions. Another possibility is to use novel 326 techniques that combine ecological, phylogenetic, and geographic information to predict 327 forbidden links and define a realized rather than a potential matrix of species interactions 
328 for large communities [76]. This latter possibility infers the strength of species interaction

329 (e.g., competition, mutualism, etc.) without the necessity of measuring fitness directly. In

330 sum, obtaining information for estimating the matrix $\boldsymbol{\beta}$ is challenging, but there are

331 techniques available to solve that limitation [75-77].

333 This three-step approach can also be combined with variation in species functional traits,

334 phylogenetic relatedness, or intra-specific variation to test a myriad of ecological questions

335 regarding the functional and phylogenetic assembly of communities (e.g. limiting

336 similarity hypothesis, Darwin's naturalization hypothesis) [4, 78]. Moreover, measuring

337 emergent properties of the community such as biomass or food production would allow

338 linking the mechanisms of biodiversity maintenance to ecosystem functioning (e.g.

339 biodiversity insurance hypothesis, biodiversity-complementarity hypothesis) [79, 80]. For

340 instance, experimental assemblages varying plant and flower morphology and pollinators'

341 body size can allow testing the role of species traits in providing higher food production

342 yields [81] by the effects of plant and animal traits on niche and fitness differences (see

343 Ref. [82] for details).

\section{Concluding remarks}

346 The integration of niche and network theories provides a natural pathway to obtain a

347 deeper understanding of the role of species interactions in modulating species coexistence.

348 Here, we show that this integration is straightforward thanks to the strong parallelism of

349 ecological concepts, complementary approaches, and associated mathematical tools found

350 across these two research areas. The emergent property of this integration is the

351 consideration that diversity within ecological communities is maintained when species

352 interactions creates a coexistence space that accommodates the differences in fitness

353 between species. Importantly, we have provided a methodological framework readily 
354 available to investigate how the strength of mutualistic, antagonistic, and competitive

355 interactions across trophic levels promote species coexistence in multi-trophic networks

356 and variable environments. The key limitation we face now is the empirical

357 parameterization of the interaction matrix, which summarizes the structure of species

358 interactions across trophic levels. It should be no surprise that applying the integration of

359 niche and network theory to experimental and observational approaches can be

360 challenging, but we have provided a guideline to accomplish this aim. While this is not an

361 easy task, the benefits can be unlimited.

\section{Acknowledgements}

364 Comments from three anonymous reviewers greatly improve the quality of this paper,

365 which is the result of a collaborative project funded by the Spanish Ministry of Economy 366 and Competitiveness (LINCX, CGL2014-61590-EXP). OG acknowledges postdoctoral

367 financial support provided by the Spanish Ministry of Economy and Competitiveness (JCI-

368 2012-12061) and by the European Union Horizon 2020 research and innovation program

369 under the Marie Sklodowska-Curie grant agreement No 661118-BioFUNC. SS also

370 acknowledges support from MIT Research Committee Funds and the Mitsui Chair. We

371 finally thank Phylopic.com and Melissa Broussard for providing silhouette images of 372 plants and pollinators.

\section{Author contributions}

375 All authors contribute equally to the paper. 


\section{References}

377 1. Darwin, C. (1859) The origin of the species, Modern Library.

2. Grinnell, J. (1917) The niche-relationships of the California Thrasher. The Auk 34 (4), 427433.

3. Elton, C. (1946) Competition and the structure of ecological communities. The Journal of 381 Animal Ecology, 54-68.

4. Macarthur, R. and Levins, R. (1967) The limiting similarity, convergence, and divergence of coexisting species. The American Naturalist 101 (921), 377-385.

5. May, R.M. and Mac Arthur, R.H. (1972) Niche overlap as a function of environmental variability. Proceedings of the National Academy of Sciences 69 (5), 1109-1113.

6. Hutchinson, G.E. (1961) The paradox of the plankton. American Naturalist, 137-145.

7. Abrams, P. (1983) The theory of limiting similarity. Annual review of ecology and systematics $14,359-376$.

8. Tilman, D. (1982) Resource competition and community structure, Princeton University

390 Press.

391 9. McKane, R.B. et al. (2002) Resource-based niches provide a basis for plant species diversity 392 and dominance in arctic tundra. Nature 415 (6867), 68-71.

393 10. Hautier, Y. et al. (2009) Competition for Light Causes Plant Biodiversity Loss After 394 Eutrophication. Science 324 (5927), 636-638.

395 11. Pauw, A. (2013) Can pollination niches facilitate plant coexistence? Trends in ecology \& 396 evolution $28(1), 30-37$.

397 12. Bennett, J.A. et al. (2017) Plant-soil feedbacks and mycorrhizal type influence temperate 398 forest population dynamics. Science 355 (6321), 181-184.

399 13. Runquist, R.B. and Stanton, M.L. (2013) Asymmetric and frequency-dependent pollinator400 mediated interactions may influence competitive displacement in two vernal pool plants.

401 Ecology letters $16(2), 183-190$. 
14. Howe, H.F. and Estabrook, G.F. (1977) On intraspecific competition for avian dispersers in tropical trees. The American Naturalist 111 (981), 817-832.

15. Polis, G.A. et al. (1989) The ecology and evolution of intraguild predation: potential

405 competitors that eat each other. Annual review of ecology and systematics 20 (1), 297-330.

406 16. Abrams, P.A. et al. (1998) Apparent competition or apparent mutualism? Shared predation 407 when populations cycle. Ecology 79 (1), 201-212.

408 17. Pacala, S. and Crawley, M. (1992) Herbivores and plant diversity. American Naturalist, $409 \quad 243-260$.

410 18. Parker, I.M. et al. (2015) Phylogenetic structure and host abundance drive disease pressure 411 in communities. Nature 520 (7548), 542-544.

412 19. Gilbert, G.S. and Webb, C.O. (2007) Phylogenetic signal in plant pathogen-host range.

413 Proceedings of the National Academy of Sciences 104 (12), 4979-4983.

414 20. Bertness, M.D. and Callaway, R. (1994) Positive interactions in communities. Trends in 415 ecology \& evolution 9 (5), 191-193.

416 21. Callaway, R.M. (1995) Positive interactions among plants. The Botanical Review 61 (4), $417 \quad 306-349$.

418 22. Holt, R.D. (1977) Predation, apparent competition, and the structure of prey communities.

419 Theoretical population biology $12(2), 197-229$.

420 23. Holt, R.D. (1984) Spatial heterogeneity, indirect interactions, and the coexistence of prey 421 species. American Naturalist, 377-406.

422 24. Chesson, P. and Kuang, J.J. (2008) The interaction between predation and competition. $423 \quad$ Nature 456 (7219), 235-238.

424 25. Chesson, P. (2013) Species competition and predation. In Ecological systems, pp. 223-256, 425 Springer.

426 26. Chase, J.M. et al. (2002) The interaction between predation and competition: a review and 427 synthesis. Ecology Letters 5 (2), 302-315. 
27. Deraison, H. et al. (2015) Functional trait diversity across trophic levels determines herbivore impact on plant community biomass. Ecology letters 18 (12), 1346-1355. 28. Soliveres, S. et al. (2016) Biodiversity at multiple trophic levels is needed for ecosystem 431 multifunctionality. Nature 536, 456.

432 29. Bascompte, J. and Solé, R.V. (1995) Rethinking complexity: modelling spatiotemporal 433 dynamics in ecology. Trends in Ecology \& Evolution 10 (9), 361-366.

434 30. Harfoot, M.B. et al. (2014) Emergent global patterns of ecosystem structure and function 435 from a mechanistic general ecosystem model. PLoS biology 12 (4), e1001841.

436 31. Jordano, P. et al. (2003) Invariant properties in coevolutionary networks of plant-animal 437 interactions. Ecology letters $6(1), 69-81$.

438 32. Bascompte, J. and Jordano, P. (2007) Plant-animal mutualistic networks: the architecture of 439 biodiversity. Annu. Rev. Ecol. Evol. Syst. 38, 567-593.

440 33. Bastolla, U. et al. (2009) The architecture of mutualistic networks minimizes competition 441 and increases biodiversity. Nature 458 (7241), 1018-1020.

442 34. Pocock, M.J. et al. (2012) The robustness and restoration of a network of ecological 443 networks. Science 335 (6071), 973-977.

444 35. Rohr, R.P. et al. (2014) On the structural stability of mutualistic systems. Science 345 445 (6195), 1253497.

446 36. Lafferty, K.D. et al. (2008) Parasites in food webs: the ultimate missing links. Ecology 447 letters $11(6), 533-546$.

448 37. Allesina, S. and Pascual, M. (2008) Network structure, predator-prey modules, and 449 stability in large food webs. Theoretical Ecology 1 (1), 55-64.

450 38. May, R.M. and Leonard, W.J. (1975) Nonlinear aspects of competition between three 451 species. SIAM Journal on Applied Mathematics 29 (2), 243-253.

452 39. Chase, J.M. and Leibold, M.A. (2003) Ecological niches: linking classical and 453 contemporary approaches, University of Chicago Press. 
40. Case, T.J. (1999) Illustrated Guide to Theoretical Ecology. Ecology 80 (8), 2848-2848.

41. Chesson, P. (1990) MacArthur's consumer-resource model. Theoretical Population Biology $37(1), 26-38$.

42. Kuang, J.J. and Chesson, P. (2009) Coexistence of annual plants: generalist seed predation weakens the storage effect. Ecology 90 (1), 170-182.

43. Godoy, O. and Levine, J.M. (2014) Phenology effects on invasion success: insights from coupling field experiments to coexistence theory. Ecology 95 (3), 726-736.

44. Hutchinson, G.E. (1957) Concluding remarks. Cold spring harbor symposium on quantitative biology 22, 415-427.

45. Diamond, J.M. (1975) Assembly of species communities. In Ecology and evolution of communities (Cody, M. and Diamond, J.M. eds), pp. 342-444, Harvard University Press. 46. Violle, C. et al. (2011) Phylogenetic limiting similarity and competitive exclusion. Ecology letters $14(8), 782-787$.

47. Pacala, S.W. and Tilman, D. (1994) Limiting similarity in mechanistic and spatial models of plant competition in heterogeneous environments. American naturalist, 222-257.

48. Chesson, P. (2000) Mechanisms of maintenance of species diversity. Annual review of Ecology and Systematics 31 (1), 343-366.

49. Adler, P.B. et al. (2007) A niche for neutrality. Ecology letters 10 (2), 95-104.

50. Kraft, N.J.B. et al. (2015) Plant functional traits and the multidimensional nature of species coexistence. Proceedings of the National Academy of Sciences 112 (3), 797-802.

51. Leibold, M.A. and McPeek, M.A. (2006) Coexistence of the niche and neutral perspectives in community ecology. Ecology 87 (6), 1399-1410.

52. Roberts, A. (1974) The stability of a feasible random ecosystem. Nature 251 (5476), 607608.

53. Logofet, D.O. (2005) Stronger-than-Lyapunov notions of matrix stability, or how "flowers" help solve problems in mathematical ecology. Linear algebra and its applications 398, 75-100. 
54. Hofbauer, J. and Sigmund, K. (1998) Evolutionary games and population dynamics,

481 Cambridge university press.

482 55. Saavedra, S. et al. (2016) Seasonal species interactions minimize the impact of species 483 turnover on the likelihood of community persistence. Ecology 97 (4), 865-873.

484 56. Kéfi, S. et al. (2016) How structured is the entangled bank? The surprisingly simple 485 organization of multiplex ecological networks leads to increased persistence and resilience. 486 PLoS biology $14(8)$, e1002527.

487 57. Levine, J.M. and HilleRisLambers, J. (2009) The importance of niches for the maintenance 488 of species diversity. Nature 461 (7261), 254-257.

489 58. Narwani, A. et al. (2013) Experimental evidence that evolutionary relatedness does not 490 affect the ecological mechanisms of coexistence in freshwater green algae. Ecology letters 16 $491 \quad(11), 1373-1381$.

59. Godoy, O. et al. (2017) Intransitivity is infrequent and fails to promote annual plant

493 coexistence without pairwise niche differences. Ecology 98 (5), 1193-1200.

494 60. Germain, R.M. et al. (2016) Species coexistence: macroevolutionary relationships and the 495 contingency of historical interactions. Proc. R. Soc. B 283 (1827), 20160047.

496 61. Hart, S.P. et al. (2016) How variation between individuals affects species coexistence.

497 Ecology letters $19(8), 825-838$.

498 62. Turcotte, M.M. and Levine, J.M. (2016) Phenotypic plasticity and species coexistence.

499 Trends in ecology \& evolution 31 (10), 803-813.

500 63. Siepielski, A.M. et al. (2011) Signature of ecological partitioning in the maintenance of 501 damselfly diversity. Journal of Animal Ecology 80 (6), 1163-1173.

502 64. Chu, C. and Adler, P.B. (2015) Large niche differences emerge at the recruitment stage to 503 stabilize grassland coexistence. Ecological Monographs 85 (3), 373-392.

504 65. Ives, A. et al. (2003) Estimating community stability and ecological interactions from timeseries data. Ecological Monographs 73 (2), 301-330. 
66. Kunstler, G. et al. (2016) Plant functional traits have globally consistent effects on competition. Nature 529 (7585), 204-207. 67. Angert, A.L. et al. (2009) Functional tradeoffs determine species coexistence via the 509 storage effect. Proceedings of the National Academy of Sciences 106 (28), 11641-11645. 510 68. Coux, C. et al. (2016) Linking species functional roles to their network roles. Ecology 511 Letters $19(7), 762-770$.

512 69. Buchmann, S.L. (1987) The ecology of oil flowers and their bees. Annual Review of 513 Ecology and Systematics 18 (1), 343-369.

514 70. Benadi, G. et al. (2014) Specialization and phenological synchrony of plant-pollinator 515 interactions along an altitudinal gradient. Journal of Animal Ecology 83 (3), 639-650.

516 71. Saavedra, S. et al. (2017) A structural approach for understanding multispecies coexistence.

517 Ecological Monographs 87 (3), 470-486.

518 72. Mordecai, E.A. et al. (2016) The role of competition-colonization tradeoffs and spatial 519 heterogeneity in promoting trematode coexistence. Ecology 97 (6), 1484-1496.

520 73. Pilosof, S. et al. (2017) The multilayer nature of ecological networks. Nature Ecology \& 521 Evolution 1, 0101.

522 74. Fründ, J. et al. (2013) Bee diversity effects on pollination depend on functional 523 complementarity and niche shifts. Ecology 94 (9), 2042-2054.

524 75. Tack, A.J. et al. (2011) Can we predict indirect interactions from quantitative food webs?525 an experimental approach. Journal of Animal Ecology 80 (1), 108-118.

526 76. Morales-Castilla, I. et al. (2015) Inferring biotic interactions from proxies. Trends in 527 ecology \& evolution 30 (6), 347-356.

528 77. Bartomeus, I. et al. (2016) A common framework for identifying linkage rules across 529 different types of interactions. Functional Ecology 30 (12), 1894-1903.

530 78. Duncan, R.P. and Williams, P.A. (2002) Ecology: Darwin's naturalization hypothesis 531 challenged. Nature 417 (6889), 608-609. 

biodiversity experiments. Nature 412 (6842), 72-76.

80. Lawton, J.H. and Brown, V.K. (1994) Redundancy in ecosystems. In Biodiversity and ecosystem function, pp. 255-270, Springer.

536 81. Garibaldi, L.A. et al. (2015) Trait matching of flower visitors and crops predicts fruit set 537 better than trait diversity. Journal of Applied Ecology 52 (6), 1436-1444. 82. Carroll, I.T. et al. (2011) Niche and fitness differences relate the maintenance of diversity to ecosystem function. Ecology 92 (5), 1157-1165.

540 83. Pashalidou, F.G. et al. (2015) Early herbivore alert matters: plant-mediated effects of egg 541 deposition on higher trophic levels benefit plant fitness. Ecology letters 18 (9), 927-936.

542 84. Rueda, M. et al. (2013) Contrasting impacts of different-sized herbivores on species 543 richness of Mediterranean annual pastures differing in primary productivity. Oecologia 172 (2), $544 \quad 449-459$.

545 85. Louthan, A.M. et al. (2013) Climatic stress mediates the impacts of herbivory on plant 546 population structure and components of individual fitness. Journal of Ecology 101 (4), 1074$547 \quad 1083$.

548 86. Bever, J.D. et al. (2015) Maintenance of plant species diversity by pathogens. Annual 549 review of ecology, evolution, and systematics $46,305-325$.

550 87. Venner, S. et al. (2011) Coexistence of insect species competing for a pulsed resource: 551 toward a unified theory of biodiversity in fluctuating environments. PLoS One 6 (3), e18039. 552 88. Reitz, S.R. and Trumble, J.T. (2002) Competitive displacement among insects and 553 arachnids. Annual review of entomology 47 (1), 435-465.

554 89. Mattila, H.R. and Seeley, T.D. (2007) Genetic diversity in honey bee colonies enhances 555 productivity and fitness. Science 317 (5836), 362-364.

556 90. Jongejan, F. and Uilenberg, G. (2004) The global importance of ticks. Parasitology 129 $557 \quad(\mathrm{~S} 1), \mathrm{S} 3-\mathrm{S} 14$. 
91. Moore, J.W. and Schindler, D.E. (2010) Spawning salmon and the phenology of emergence

559 in stream insects. Proceedings of the Royal Society of London B: Biological Sciences,

$560 \quad$ rspb20092342.

561 92. Abzhanov, A. et al. (2004) Bmp4 and morphological variation of beaks in Darwin's

562 finches. Science 305 (5689), 1462-1465.

563 93. Creel, S. and Creel, N.M. (1996) Limitation of African wild dogs by competition with

564 larger carnivores. Conservation Biology 10 (2), 526-538.

565 94. Voeten, M.M. and Prins, H.H. (1999) Resource partitioning between sympatric wild and

566 domestic herbivores in the Tarangire region of Tanzania. Oecologia 120 (2), 287-294.

567 95. Mishra, C. et al. (2004) Competition between domestic livestock and wild bharal Pseudois

568 nayaur in the Indian Trans-Himalaya. Journal of Applied Ecology 41 (2), 344-354.

569 96. Askew, R.R. (1971) Parasitic insects. Parasitic insects.

570 97. Cobey, S. and Lipsitch, M. (2012) Niche and neutral effects of acquired immunity permit

571 coexistence of pneumococcal serotypes. Science 335 (6074), 1376-1380.

572 98. Cenci, S. et al. (2018) Estimating the effect of the reorganization of interactions on the

573 adaptability of species to changing environments. Journal of theoretical biology 437, 115-125.

574 99. Svirezhev, I.U. and Logofet, D.O. (1978) The stability of biological communities.

575 100. Morris, W.F. et al. (2010) Benefit and cost curves for typical pollination mutualisms.

576 Ecology $91(5), 1276-1285$. 


\section{Trophic level}

plant - plant

\section{Evidences of niche and fitness differences}

Niche: Spatial segregation, phenology, or plant morphology differences reduce niche overlap.

Fitness: Species ability to draw down common limiting resources determines species fitness.

plant-insect

Niche: Fragmented evidences suggest that differences in pollinators can stabilize plant coexistence.

$[11,70,83]$

Fitness: Herbivorous insects and their network of hyperparasitoids can significantly affect plant fitness.

plant-vertebrate

Niche and fitness: Interactive effects between abiotic stress, tolerance to herbivory and herbivore body size

determine plant abundances and richness.

plant - fungi

pathogens and through pathogen specialization.

Fitness: Low specificity of fungal pathogens determines local abundance of plant species in tropical forest.

herbivorous insects. Time partitioning for predating acorns determine niche differences within a guild of 
phytophagous insects.

Fitness: Searching ability, female fecundity, and resource degradation and preemption determine fitness

differences among parasitoids, phytophagous insects and arachnids.

insect - plant Niche: Wild bees specialize in their floral reward including nectar, pollen, pollen resins, volatiles, lipids, and

waxes.

Fitness: Foraging rates and food storage determine fitness differences (i.e. drone production, winter survival) among genetically diverse honey-bees colonies.

insect - vertebrate Tick habitat differs greatly among species from rodent burrows, caves to bird nest.

Fitness: The timing and duration of aquatic insect emergence is regulated by temporal variation in salmon density.

vertebrate - vertebrate Niche: Differences in bill shape and body size stabilize coexistence between birds by the use of different resources.

Fitness: Intraguild predation of large carnivores on African wild dogs reduces its population size

\begin{tabular}{ll}
\hline plant - vertebrate & Niche: Strong overlap of dietary requirements between wild and domestic herbivores. \\
\hline insect - vertebrate & Niche: Vertebrates differ in the number and specificity of their parasitic insects. \\
& Fitness: Ticks reduce offspring and increase mortality in a wide variety of animal including birds, lizards and \\
& mammals.
\end{tabular}


Fitness: Competition-colonization trade-offs determines trematodes fitness.

alga - alga

Niche and fitness: Phylogeny relatedness does not predict competitive outcomes between fresh water algae.

bacteria - vertebra

Niche: Specific immunity of Streptococcus pneumoniae serotypes stabilizes coexistence.

Fitness: Acquired immunity to non-capsular antigens determines serotypes fitness. 
Domain of niche theory studies

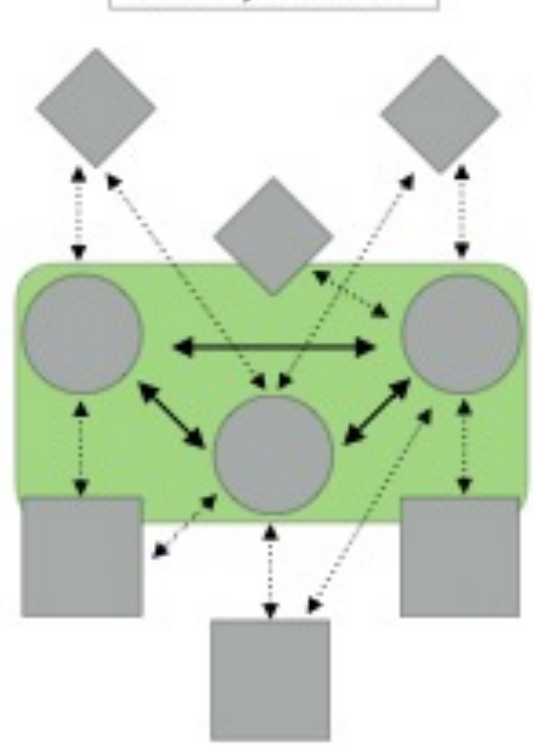

Domain of network theory studies

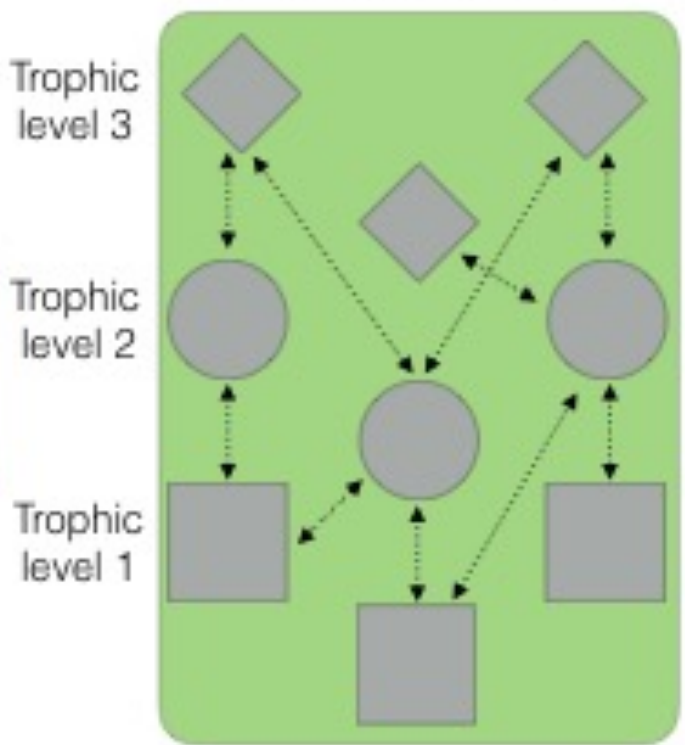

584 Figure 1: Research domains of niche and network theories. Niche theory (left side) has

585 been successful in incorporating the effect of direct and indirect interactions within and

586 between trophic levels (denote by arrows in black) on determining species coexistence

587 within a single trophic level (light green rectangle) [7, 16, 22, 24]. However, niche studies

588 have not addressed how these direct and indirect trophic interactions modulate coexistence

589 across trophic levels. In contrast, this task has been addressed by network research (right

590 side). While the area of study is bigger (green rectangle), network studies have not

591 considered the structure of interactions within trophic levels (no solid lines present). By

592 integrating niche and network theories we can start considering explicitly and

593 simultaneously species interactions across trophic levels and their role (feed-backs) in

594 modulating species coexistence. Note that arrows are double headed indicating the

595 existence of such feedbacks. Solid and dashed arrows indicate whether the interaction is

596 within or between trophic levels, respectively. 


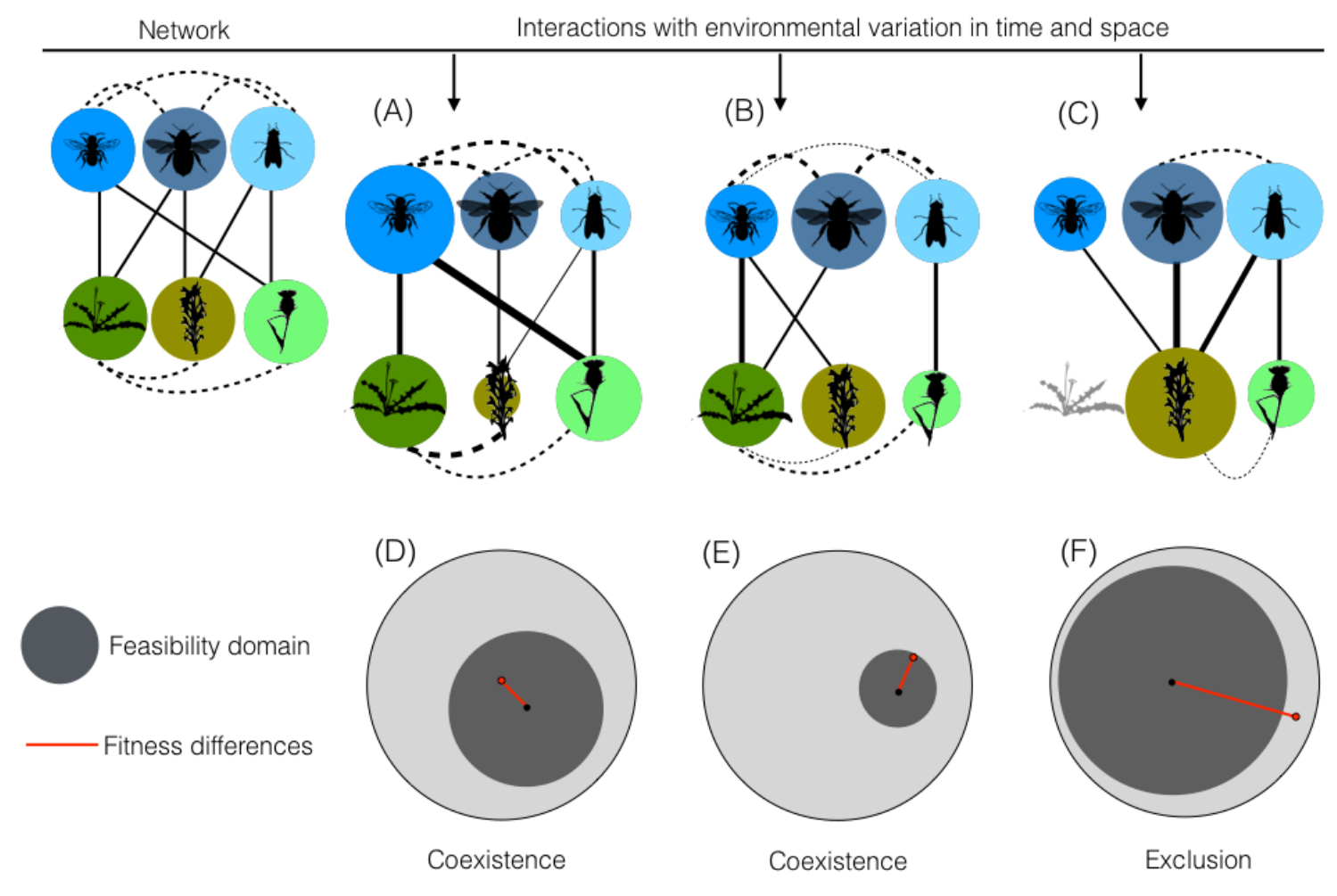

Figure 2: Effects of species' intrinsic properties and network structure on species coexistence. Species traits such as body size, phenological timing, or feeding preferences

600 interact with environmental variations in space and time to determine 1) network structure 601 and 2) species' fitness. Dashed lines represent the strength of species interactions within 602 trophic levels and solid lines represent the same across trophic levels. Obtaining 603 information on how such trait-environment interactions modified these two elements in 604 ecological communities remains fundamental to predict the consequences of species 605 interactions for the maintenance of diversity [98]. Consider a hypothetical case of a plant606 pollinator system in which environmental variation modifies these two elements in three 607 different ways (Panels A, B, and C). The size of the circles denotes the realized species' 608 fitness, which arises as a combination of species interactions and their demographic 609 parameters (plants in green and insects in blue). Additionally, we have learned from the 610 integration of niche and network theories that the structure of species interactions within 611 and between trophic levels renders the feasibility domain (Here represented in two 
612 dimensions for simplicity; dark gray area in Panels D, E, and F). Note that each network

613 structure gives a different size of feasibility domain. In principle, the larger the feasibility

614 domain, the more likely species coexist as it allows for a larger combination of fitness

615 differences (see F feasibility domain compare to D and E cases). However, it is paramount

616 to point out that even with a large feasibility domain, species may not coexist if the

617 position of the vector containing species' fitness (red line) falls outside the feasibility

618 domain (i.e., falls within the light gray area) [71]. This is the case of F where one plant

619 species is excluded. Conversely, the system can be maintained despite showing a smaller

620 feasibility region if the vector of species' fitness falls within the feasibility domain (cases

$621 \mathrm{D}$ and E). Therefore, the take-home message is that coexistence occurs when species

622 interactions creates a feasibility domain compatible with the observed fitness differences.

623 Recall that fitness differences are measured as the distance between the center of the

624 feasibility domain and the position of the vector containing species fitness (red line).

625 Importantly, systems with low fitness differences may face larger perturbations. For

626 instance, species can coexist in case E but it can be less resistant to perturbations compared

627 to case D given that the position of the vector of species' fitness is close to the exclusion

628 region. 
631 For a pair of species in competition, the coexistence conditions according to niche theory are defined by:

$$
\underbrace{\sqrt{\frac{\alpha_{11} \alpha_{22}}{\alpha_{12} \alpha_{21}}}}_{\text {(1-Niche difference) }^{-1}}>\underbrace{\frac{r_{1}}{r_{2}} \sqrt{\frac{\alpha_{22} \alpha_{21}}{\alpha_{11} \alpha_{12}}}}_{\text {Fitness difference }}>\underbrace{\sqrt{\frac{\alpha_{12} \alpha_{21}}{\alpha_{11} \alpha_{22}}}}_{\text {1-Niche difference }}
$$

634 where $r_{1}>0$ corresponds to the intrinsic growth rate (demographic parameter) of species 1 , 635 and $\alpha_{12}>0$ represents the competitive per capita effect of species 2 on the per capita growth 636 rate of species 1 . This equation states that the fitness difference (i.e., the ratio between 637 intrinsic growth rates modulated by what is known as the competitive response ratio) of the 638 two species has to fall between a lower and an upper bound computed from the niche 639 difference (i.e., range of values defined by the ratio between inter and intra-specific 640 competition). Note that these inequalities can be also simply written as $\alpha_{11} / \alpha_{21}>r_{1} / r_{2}>$ $641 \alpha_{21} / \alpha_{22}$. Moreover, such inequalities have also to assume that the niche difference is smaller 642 than one, i.e.,

$$
\alpha_{12} \alpha_{21}<\alpha_{11} \alpha_{22}
$$

644 which guarantees that the equilibrium point is dynamically stable (the system returns to its 645 original equilibrium point after a pulse perturbation) in a Lotka-Volterra competition model 646 of the form:

$$
\left\{\begin{array}{l}
\frac{d N_{1}}{d t}=N_{1}\left(r_{1}-\alpha_{11} N_{1}-\alpha_{12} N_{2}\right) \\
\frac{d N_{2}}{d t}=N_{2}\left(r_{2}-\alpha_{21} N_{1}-\alpha_{22} N_{2}\right)
\end{array}\right.
$$

648 where $N_{l}$ and $N_{2}$ correspond to the abundance of species 1 and 2, respectively. Note that, 649 the inequalities in Equation 1 correspond to an equilibrium point called feasible because all 650 species have positive abundances (i.e., $N_{1}^{*}>0$ and $N_{2}^{*}>0$ ) $[35,52-54,99]$. In contrast, the 

inequality of Equation 2 only grants the dynamical stability (in fact in that specific case the

652 global stability) by having intra-specific competition stronger than interspecific

653 competition. Note that feasibility is a necessary condition for species persistence in a

654 Lotka-Volterra model [54].

655

656 Let us explain how dynamical stability and feasibility conditions arise in multi-trophic

657 systems by taking as an example a two-trophic level system describing the mutualistic

658 interactions between a set of plants (P) and a set of pollinators (A). Note that similar

659 conclusions are obtained by considering antagonist interactions such as a prey-predator

660 system. This mutualistic system can be described by the following set of dynamical

661 equations:

662

$$
\left\{\begin{array}{l}
\frac{d P_{i}}{d t}=P_{i}\left(r_{i}^{(P)}-\sum_{j} \alpha_{i j}^{(P)} P_{j}+\sum_{j} \gamma_{i j}^{(P)} A_{j}\right) \\
\frac{d A_{i}}{d t}=A_{i}\left(r_{i}^{(A)}-\sum_{j} \alpha_{i j}^{(A)} A_{j}+\sum_{j} \gamma_{i j}^{(A)} P_{j}\right)
\end{array}\right.
$$

664 where the variables $P_{i}$ and $A_{i}$ denote the abundance of plant and animal species i, respectively. The parameters of this mutualistic model correspond to the values describing

666 intrinsic growth rates $\left(r_{i}\right)$, within-guild competition $\left(\alpha_{i j}>0\right)$, and the benefit received via 667 mutualistic interactions between trophic levels $\left(\gamma_{i j}>0\right)$. All these interaction strengths can,

668 in turn, be embedded in a two-by-two block matrix $\boldsymbol{\beta}=\left[\begin{array}{cc}\boldsymbol{a}^{(\mathbf{P})} & -\gamma^{(\mathbf{P})} \\ -\gamma^{(\mathbf{A})} & \boldsymbol{a}^{(\mathbf{A})}\end{array}\right]$. The conditions for

669 feasibility depend on both the species interactions defined by $\beta$ and the demographic

670 parameters of species $r$ (analogous to equation (1) above) [71]. Note that the conditions for 671 dynamical stability are more complex [40]. Indeed, several meaningful notions of stability 672 have been defined in ecology, such as Volterra-dissipative, D-stability, sign-stability, and 
673 local stability. Sign-stability, Volterra-dissipative, and D- stability are only determined by 674 the interaction matrix $\boldsymbol{\beta}$. Sign-stability has the property of granting global stability only on 675 the description of who eats whom and not on the strength of the trophic interactions.

676 Volterra-dissipative implies the global stability of a feasible equilibrium, while D-stability 677 grants only local stability. Finally, local stability involves also the equilibrium densities and 678 therefore the intrinsic growth rates. The relations among these notions of stability (and 679 more) are well represented by Logofet's flower [53]. 


\section{Box 2: Emerging properties of the integration of niche and network theory}

For multi-trophic dynamical systems of the general form $d N_{i} / d t=N_{i} f_{i}(\mathbf{N})$, an $n \times n$ block matrix emerges for describing species interaction across $\mathrm{n}$ trophic levels:

$$
\beta=\left[\begin{array}{cccc}
\alpha_{1} & \gamma_{12} & \cdots & \gamma_{1 n} \\
\gamma_{21} & \alpha_{2} & \cdots & \gamma_{2 n} \\
\vdots & \vdots & \ddots & \vdots \\
\gamma_{n 1} & \gamma_{n 2} & \cdots & \alpha_{n}
\end{array}\right]
$$

where the diagonal blocks $\left(\alpha_{i}\right)$ correspond to the within-trophic level (i) interactions (i.e. competition, intraguild predation, facilitation) and the other blocks $\left(\gamma_{i j}\right)$ represent the betweentrophic level interactions (effect of trophic level $\mathrm{j}$ on $\mathrm{i}$ in the form of mutualism or antagonism interactions). As $\boldsymbol{\beta}$ is a block matrix, each element of the matrix represents a submatrix of species interaction. For instance, $\boldsymbol{\alpha}_{l}$ is a matrix describing all species interaction within the trophic level 1, and $\gamma_{12}$ is another matrix describing all interactive effects of species from the trophic level 2 on species from the trophic level 1.

Stable coexistence of all species $\left(N_{i}^{*}>0\right)$ across trophic levels depends on whether this interaction matrix $\boldsymbol{\beta}$ and the demographic parameters $r_{i}$ satisfies together both the stability and feasibility conditions $[53,54,71]$. There are different classes of dynamical stability. For instance, local stability is the property of the system to return to the equilibrium point after a small pulse perturbation (changes in species abundances), whereas global stability is concerned with external perturbations of any given magnitude converging to the same equilibrium point. Each class demands specific properties to be fulfilled by the interaction matrix $\beta$ in combination with the species demographic parameters $r_{i}[53,71]$, and which class of stability should be studied depends on both the research question and knowledge about the system. The feasibility of a multi-trophic system corresponds to the conditions allowing all species to have positive abundances, which also depends on both the interaction matrix $\beta$ and the demographic parameters $r_{i}[35,52-54,71]$. The figure below illustrates the conditions of feasibility in a 
three-species system. The green area on the sphere represents the range of demographic parameters leading to feasibility given the interaction strengths matrix. To some extent, Fig. i of the extension of modern coexistence theory to multispecies coexistence; the border of the

707 green area is the multispecies analogous of the fitness and niche difference inequality (Box 1, 708 Equation 1) that applies to species pairs only.

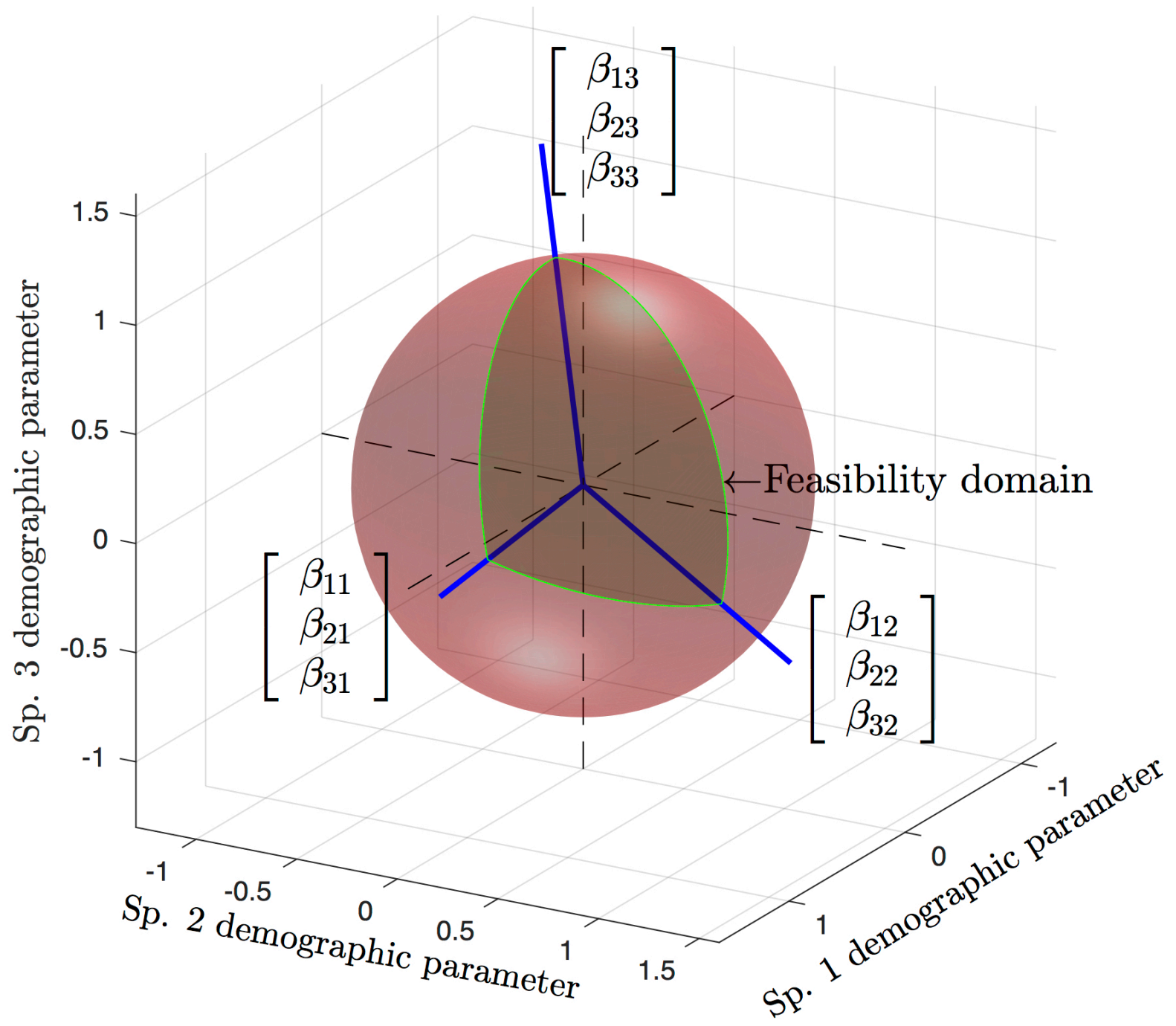

710 Figure i: Illustration of the feasibility domain for a multi-trophic system. The figure shows the

711 normalized domain of demographic parameters (feasibility domain relative to the unit sphere)

712 that a two-trophic system (e.g., two pollinators and one plant) can theoretically have to be

713 compatible with all species having positive abundances. This normalized feasibility domain 
714 (green spherical triangle) is constrained by the intra- and interspecific interaction matrix ( $\boldsymbol{\beta})$.

715 The columns of the interaction matrix (e.g. $\left.\left[\beta_{11}, \beta_{12}, \beta_{13}\right]\right)$ give the boundaries of the feasibility

716 domain (three blue lines). The larger this volume is, the larger the set of demographic values

717 compatible with feasibility, and the larger the likelihood of species coexistence across trophic 718 levels $[55,71]$. 
721 Our approach to evaluate how between trophic interactions drive niche and fitness differences within trophic levels and vice versa involves three steps.

Step. 1: Depart from a relatively simple system of equations. Here it is composed of two annual population models describing changes in population size with time in plants (seeds, $P_{i, t+1}$ ) and pollinators (eggs, $A_{i, t+1}$ ). Both models are mirror images including an equal number of parameters with the same biological meaning,

$$
\left\{\begin{array}{l}
P_{i, t+1}=P_{i, t}\left(\left(1-g_{i}\right) s_{i}+\frac{\lambda_{i} g_{i}\left(1+\sum_{k} \gamma_{i k} A_{k, t}\right)}{1+\sum_{j} \alpha_{i j} g_{j} P_{j, t}}\right) \\
A_{i, t+1}=A_{i, t}\left(\left(1-e_{i}\right) t_{i}+\frac{v_{i} e_{i}\left(1+\sum_{k} \delta_{i k} P_{k, t}\right)}{1+\sum_{j} \theta_{i j} e_{j} A_{j, t}}\right)
\end{array},\right.
$$

731 where each model is the summation of two components. The first component describes the

732 possibility of a storage effect process, and the second component describes per capita

733 fecundity. Specifically, this second component describes how mutualisms enhances the species

734 intrinsic ability to produce off spring reduced by the competitive effects exerted by other 735 species within the same guild.

Step. 2: Estimates species vital rates. Estimate per capita growth rate in the absence of species

738 interaction (plants ( $\lambda$ 's), pollinators $\left(v^{\prime} s\right)$ ), is best described as the intercept of the statistical 739 models built for step 3 (see below). Additional efforts are needed to estimate rates of seed 740 germination ( $g$ 's) or larva survival ( $\boldsymbol{e}$ 's), and the storage effect as the survival of the species'

741 life stages that do not produce offspring within a year (e.g. soil seed bank in plants ( $s$ 's) and 742 non-reproductive adult mortality in some pollinators $\left.\left(\boldsymbol{t}^{\prime} \mathrm{s}\right)\right)$. 
744 Step. 3: Estimate species interaction matrix. To estimate species intra- and inter-specific

745 competitive interactions within trophic levels (plants ( $\boldsymbol{\alpha}$ 's), pollinators $(\boldsymbol{\theta}$ 's), the best approach

746 is to fit a series of statistical models describing for each species its per capita growth rate as a

747 function of competitor's relative abundance. For mutualistic interactions (pollinator's effect on

748 plants ( $\boldsymbol{y}^{\prime}$ 's), plants' effect on pollinators $(\boldsymbol{\delta}$ 's $\left.)\right)$, do the same but describe

749 species' per capita growth rate as a function of mutualistic's relative abundances (Fig. ii).

750

751
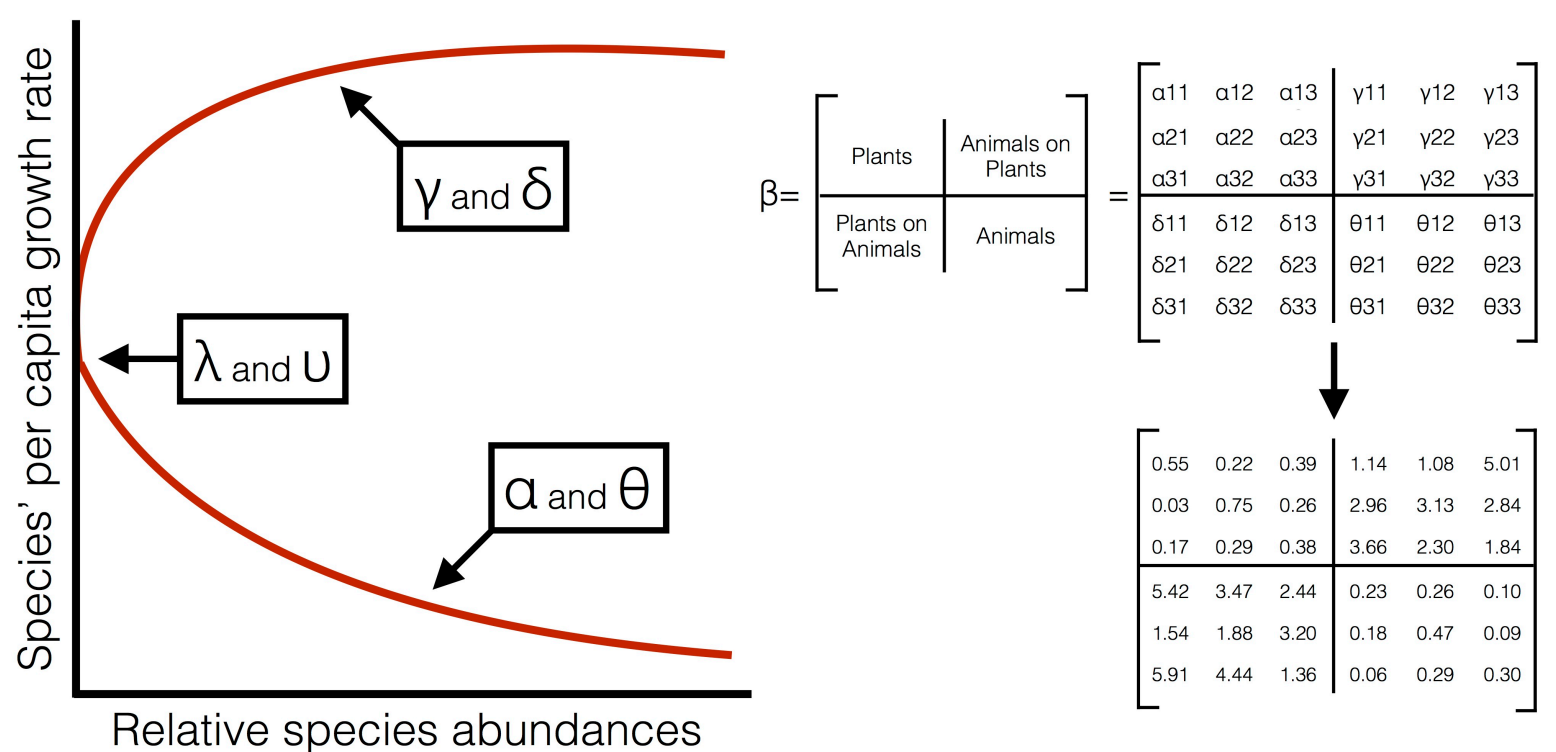

752

753 Figure ii: Competitive relationships between species including itself are expected to take a

754 negative exponential form $[59,66]$, whereas mutualistic relationship is expected to be

755 functionally saturating best described by non-inflicted curves [100]. With this information is

756 possible to then build the $\boldsymbol{\beta}$ matrix summarizing species interactions across trop 
759 Ecological network: A synthesis of biotic interaction between organisms present in a

760 community in which species represented by nodes are connected by pairwise inter-specific

761 interactions.

763 Equalizing mechanisms: Mechanisms that reduce the likelihood of species exclusion

764 within trophic levels by promoting similar fitness between competitors.

Equilibrium point: A fixed state at which species abundances are constant over time.

Dynamical stability: The property of an ecological system to return to an original equilibrium point after a pulse perturbation.

Intrinsic growth rate: The rate at which a population increases in size in the absence of density dependent regulation.

Feasibility: The property of an ecological system to hold an equilibrium point with positive abundances in all its constituent species.

Feasibility domain: The range of conditions (e.g., demographic parameters) compatible with all species having positive abundance.

Feedback: The process by which the output of a system is routed back as an input of 781 another system forming a loop. 
Fitness: The species ability to mature and produce offspring.

785 Fitness differences: According to Chesson (2000), average fitness differences

786 between species are an equalizing mechanism of species coexistence that reduce

787 competitive imbalance between competitors. In the absence of niche differences

788 determine the superior competitor in a community.

790 Multi-trophic network: A network representing patterns of multiple interaction types 791 between species including competition, mutualistic, or antagonistic relationships. Also 792 known as multiplex networks.

794 Niche: The environmental conditions and resources a species requires for living and 795 reproducing.

797 Niche differences: According to Chesson (2000), niche differences are a stabilizing 798 mechanism of species coexistence by causing intra-specific competition to exceed inter799 specific competition.

801 Per capita growth rate: The relative contribution to the population increases in size 802 per individual.

804 Species dynamics: Changes in species' population over space and time.

Trophic level: A level of organization within the food chain of an ecosystem, whose organisms obtain resources in a similar way 